\title{
Effective communication with the patient
}

\author{
R. Booker
}

ABSTRACT: The chronic and slowly progressive nature of chronic obstructive pulmonary disease (COPD) can create difficulties in effective communication between healthcare provider and patient. Such barriers, which include the personal beliefs of both caregiver and patient, need to be understood and addressed if the aims of the consultation are to be met.

Patients with COPD may feel guilty, depressed and angry about their condition. Many patients with COPD are elderly and/or from lower socio-economic groups, both of which pose challenges to the caregiver, as does the stigma the disease carries, which stems from its strong link with smoking.

Humanity, respect and people orientation are vital to good communication with COPD patients. Physicians and nurses should try to avoid patient misunderstanding and uncertainty, involve the patient in decision-making and achieve a shared understanding (concordance), as well as encourage the patient to accept responsibility for the actions agreed. This should improve management decisions made by healthcare professionals and ensure a more satisfied patient. Greater patient satisfaction may improve concordance and so bring about better patient outcomes.

KEYWORDS: Communication, concordance, consultation, inhaler technique, self-management

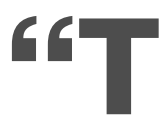
he consultation is the central act of medicine and as such it deserves to be understood" [1].

As the "central act of medicine", the consultation is the place where patients and healthcare professionals interface. It is where therapeutic relationships are formed and, when conducted well, where information is exchanged and patients' needs and concerns are recognised and addressed. Consultations are part of the "cycle of

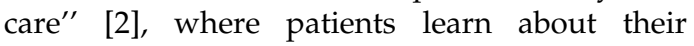
disease, come to terms with their condition and are given the ability to share in its management.

\section{BARRIERS TO COMMUNICATION The patient's experience}

Patients with chronic obstructive pulmonary disease (COPD) may experience particular difficulties that need to be understood and addressed if the aims of a consultation are to be met. COPD is a chronic and slowly progressive condition. Acceptance of any chronic disease involves a readjustment of body image and a change in expectations of the future. This can be a long process during which a host of psychological and social problems can manifest themselves [3].

As COPD is smoking related in the vast majority of cases, it is surrounded by a "blame culture" that may not exist or be apparent in other smoking-related conditions, such as coronary artery disease. Patients may be "blamed", or feel judged, for their condition by both their family and healthcare professionals, and they may also blame themselves. Guilt, anger, depression and anxiety are common emotions a patient will experience [4], which can interfere with their ability to come to terms with their condition. Anxiety, depression and negative beliefs about the disease have also been shown to hinder good self-management [5]. Patients often become defensive or apathetic; these attitudes make communication difficult and need to be recognised and understood if they are to be worked through constructively. COPD disables a person in many senses, both physically and mentally. It leads to a loss of independence and a loss of self-esteem.

Some COPD patients have difficulty accepting that they have a smoking-related disease. They feel more comfortable attributing the cause of their illness to external factors, such as their occupation or environmental pollution [6]. They might also have problems accepting the diagnosis and perhaps prefer to say that they have asthma. This can cause problems when they deal with other healthcare professionals who are not in full possession of the patient's medical history. If denial is adopted as a coping mechanism, advice about self-management may go unheeded.
CORRESPONDENCE

R. Booker

National Respiratory Training Centre Warwick CV34 4AB

UK

Fax: 441926493224

E-mail: r.booker@nrtc.org.uk 
The slowly progressive nature of COPD means that it has a greater effect on patients in middle and old age. Expectations of health may be lower in these age groups [7]. Patients may be reluctant to attend diagnostic tests and might not feel that they have a problem; typical responses are "I am just a bit prone to chest infections", or "I'm getting on a bit, so I must expect to get a bit breathless." Comorbidity is also common and can further reduce both expectations of health and what can realistically be achieved. Conversely, there are patients who are worried but who may not have their concerns adequately explored during the consultation [8]. These unexplored concerns may be those that are most relevant to the patient and may lead to dissatisfaction with the consultation, resulting in poor adherence to therapy. Patient concerns and beliefs about what happens to them now and in the future have a major influence on their behaviour [9] and can affect clinical outcomes [10].

COPD disproportionately affects lower socio-economic groups [11]. Healthcare professionals are rarely from the same socioeconomic background as their COPD patients, which can lead to difficulties in communication; neither the healthcare professional's or patient's experiences can help them to predict the other's [9]. Many COPD patients fall into a social stratum that has the least access to healthcare, little ability to effectively demand good services and is most difficult to reach with healthcare messages.

\section{The healthcare professional's experience}

Until recently there was little interest in COPD, causing it to be appropriately dubbed the "Cinderella" of respiratory diseases. There was a widely held belief that COPD was not amenable to treatment and that it was a "heart-sink" condition. In recent years, there has been greater interest in the disease, and a number of highly effective therapies and interventions are now able to improve disability and health-related quality of life. Although therapeutic nihilism can no longer be justified, it still persists, as attitudes lag behind knowledge.

Some healthcare professionals experience particular difficulties when managing patients who are unable to stop smoking [12]. Although they may have intellectual knowledge of the addictive nature of nicotine, their emotional attitudes still colour their consultations. A patient who says that smoking is their only pleasure in life may evoke little sympathy and support. Patients may feel this acutely, as many began to smoke or were actively encouraged to smoke before the risks were acknowledged and understood.

As stated above, the personal values and beliefs of the healthcare professional may enhance or impede communication. A healthcare professional who believes that one should be the master of one's own fate is less likely to be sympathetic to patients whose beliefs do not accord with their own. Healthcare professionals are human beings and factors such as mood, tiredness and distraction can affect the consultation.

\section{RESOURCES}

Although healthcare professionals are not always naturally good communicators, consultation skills can be taught and now form part of general practitioner (GP) training in the UK, although this is not widely available to nurses. In the UK, there are incentive payments for GPs who manage COPD under the General Medical Services (GMS) Contract [13]. This has encouraged greater attention to COPD in primary care, but rewards practices according to process outcomes rather than quality of results. Instead of delivering personalised patientcentred care, there is a danger that more effort will go into "ticking boxes". The task has been devolved to nurses who, though skilled at following protocols, may not have been trained in consultation.

As well as requiring support from management protocols and plans, delivery of high-quality care also requires knowledge and training. GPs are (by nature and definition) generalists and it is not possible for them to be expert in all aspects of medicine. However, it is important to patients that the doctor has a good knowledge of COPD and its management. A study by KORSCH et al. [14] found that patient satisfaction with the communication aspect of a consultation correlated highly with their satisfaction with other aspects of the consultation. Patients value the approachability of their doctor, but ideally want him or her to be both technically expert and a good communicator.

High-quality care requires time, but the belief that a good consultation is time-consuming is erroneous. Patient satisfaction with a consultation is not related to the amount of time spent, as was elegantly put by ASHER [15]: "To give a patient the impression that you could spare him an hour, and yet make him satisfied within five minutes, is an invaluable gift and of much more use than spending half an hour with him during every minute of which he is made to feel he is encroaching on your time!"

\section{REQUIREMENTS OF A GOOD CONSULTATION}

The ideal "personal specifications" for healthcare professionals who care for COPD patients are common to all aspects of healthcare. Their approach should be friendly and concerned and they should show respect for the patient's opinions, even if these differ from what they know to be in the patient's interest [16]. They should be responsive, insightful and a good listener. They should also be professional in their approach and committed to continuing to develop their own knowledge and ability.

A consultation should be patient focussed. It should achieve a shared understanding of the problem of COPD as it affects that particular patient. Patients should be actively involved in decision-making about their treatment and encouraged to accept responsibility for their management; this should result in better outcomes and a more satisfied patient. Patients prefer to be involved in the medical decision-making process, and respond better if they are [17, 18]. In a study by SMITH et al. [19], patients expressed a clear preference for joint decision-making rather than delegating the decision to the doctor or making the decision alone. Concordance, or the commitment of two parties to a shared decision, was found to be better when patients were involved in decision-making [20]. The relationship between good communication, concordance and outcomes is shown in figure 1.

There are simple ways to enhance a consultation. It is important for the doctor or nurse to greet the patient by name and to introduce themselves [16]. Eye contact and a smile will 


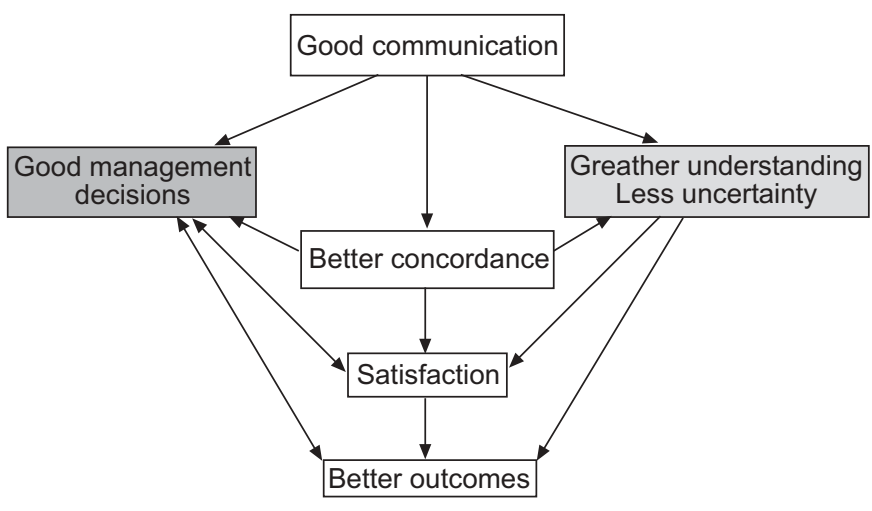

FIGURE 1. The influence of good communication between the healthcare professional ( $\square$ ) and the COPD patient ( $\square$ ) on satisfaction and outcomes. Adapted from [16] with permission from the publisher.

help to put the patient at ease. Disease-management templates and computers can be a barrier if the healthcare professional is looking at them instead of at the patient. Healthcare professionals need to be aware of both their own and the patient's non-verbal communication and must listen actively to what the patient is saying. The development of trust and a therapeutic relationship is essential for motivating patients to become involved in their care and agreeable to decisions made about it.

A good consultation involves an exchange of information that needs to be clear and truthful. Much of what is said in a consultation is forgotten [21], and in elderly COPD patients, cognitive impairment due to early dementia and/or hypoxia can be an additional barrier to comprehension [22]. Information leaflets and written instruction can be helpful, as long as they use appropriate language and the patient is able to read them. Useful information can be collected from the patient before the consultation, by means of a short questionnaire that the patient completes in the waiting room, e.g. the Breathing Problems Questionnaire [23]. Alternatively, and if the patient agrees, involving a family member or carer in the treatment plan may be helpful. It is also important to ensure patients understand and to recap and reinforce information at each subsequent consultation.

It is also helpful to discover what the patient's health beliefs and locus of control are, i.e. whether the patient is generally autonomous or more dependent, and to take these into consideration in the management plan. The autonomous patient needs to understand, remember, be motivated and act. However, not all patients want such autonomy. The different management styles are discussed further in an accompanying article within this Review [24].

\section{INHALER CHOICE AND TECHNIQUE}

Inhaler selection should form part of the consultation process for COPD patients; if the patient has been involved in this decision, they are more likely to be motivated to make it work. Consideration should also be given to lifestyle [25], and COPD patients should be encouraged to remain socially and physically active; so, for example, an inhaler that is small, discreet and easy to use is desirable (though its effectiveness in delivering drugs to the lungs must also be considered).
COPD patients can have particular difficulty with inhalers. Their inspiratory effort and flow may be limited or they may have physical, such as visual and dexterity, problems. Impaired cognitive ability may also impede a patient's ability to learn and remember the sequence of instructions necessary for successful use of an inhaler [22]. Even when inhaler technique has been learnt it often deteriorates over time; technique should be checked at every visit by a competent healthcare professional [25]. In a recent survey of 20 nurses in the Netherlands and the UK who specialise in the management of COPD patients, there was unanimous agreement that correct inhaler technique is vital to good compliance (unpublished data). Assessments of patient compliance must involve regular reviews of continuing inhaler use competence.

\section{SUMMARY}

- Humanity, respect and a people-focussed attitude are vital to good communication with patients.

- The goals of good communication are to avoid patient misunderstanding and uncertainty, improving management decisions by healthcare professionals and resulting in a more satisfied patient.

- Physicians and nurses should aim to: achieve a shared understanding with patients (concordance); involve them in decision-making; and encourage them to accept responsibility.

- Greater patient satisfaction may improve concordance and so bring about better outcomes.

\section{REFERENCES}

1 Spence J. The Purpose and Practice of Medicine. Oxford, Oxford University Press, 1960.

2 Pendleton D, Haslar J. Doctor-patient Communication. London, Academic Press, 1983.

3 Lee AM, Poole G. An application of the transactional model to the analysis of chronic illness narratives. Qual Health Res 2005; 15: 346-364.

4 van Ede L, Yzermans CJ, Brouwer HJ. Prevalence of depression in patients with chronic obstructive pulmonary disease: a systematic review. Thorax 1999; 54: 688-692.

5 Dowson CA, Town GI, Frampton C, Mulder RT. Psychopathology and illness beliefs influence COPD selfmanagement. J Psychosom Res 2004; 56: 333-340.

6 Post L, Collins C. The poorly coping COPD patient: a psychotherapeutic perspective. Int J Psychiatry Med 1981; 11: 173-182.

7 Sarkisian CA, Hays RD, Mangione CM. Do older adults expect to age successfully? The association between expectations regarding aging and beliefs regarding healthcare seeking among older adults. J Am Geriatr Soc 2002; 50: 1837-1843.

8 Tate P, Foulkes J, Neighbour R, Campion P, Field S. Assessing physicians' interpersonal skills via videotaped encounters: a new approach for the Royal College of General Practitioners Membership examination. J Health Commun 1999; 4: 143-152.

9 Pendleton D, Schofield T, Tate P, Havelock P. The new consultation: developing doctor-patient communication. Oxford, Oxford University Press, 2003. 
10 Rutter BM. The prognostic significance of psychological factors in the management of chronic bronchitis. Psychol Med 1979; 9: 63-70.

11 Prescott E, Lange P, Vestbo J. Socioeconomic status, lung function and admission to hospital for COPD: results from the Copenhagen City Heart Study. Eur Respir J 1999; 13: 1109-1114.

12 Coleman T, Cheater F, Murphy E. Qualitative study investigating the process of giving anti-smoking advice in general practice. Patient Educ Couns 2004; 52: 159-163.

13 National Health Service Confederation, British Medical Association. Investigating in general practice: the new GMS contract. London, National Health Service Confederation, British Medical Association, 2003.

14 Korsch BM, Gozzi EK, Francis V. Gaps in doctor-patient communication. 1. Doctor-patient interaction and patient satisfaction. Pediatrics 1968; 42: 855-871.

15 Asher R. Talk, tact and treatment. In: Jones FA, ed. Richard Asher talking sense. London, Pitman Medical, 1972; pp. 111-118.

16 Fallowfield L. The ideal consultation. Br J Hosp Med 1992; 47: 364-367.

17 Bourbeau J, Nault D, Dang-Tan T. Self-management and behaviour modification in COPD. Patient Educ Couns 2004; 52: $271-277$.
18 Worth $H$, Dhein $Y$. Does patient education modify behaviour in the management of COPD? Patient Educ Couns 2004; 52: 267-270.

19 Smith D, Garko M, Bennett K, Irwin H, Schofield T. Patient preferences for delegation and participation: cross-national suport for mutuality. Aus J Commun 1994; 21: 86-108.

20 Brody DS, Miller SM, Lerman CE, Smith DG, Caputo GC. Patient perception of involvement in medical care: relationship to illness attitudes and outcomes. J Gen Intern Med 1989; 4: 506-511.

21 Ley P. Memory for medical information. Br J Soc Clin Psychol 1979; 18: 245-255.

22 Allen SC, Ragab S. Ability to learn inhaler technique in relation to cognitive scores and tests of praxis in old age. Postgrad Med J 2002; 78: 37-39.

23 Hyland ME, Bott J, Singh S, Kenyon CA. Domains, constructs and the development of the breathing problems questionnaire. Qual Life Res 1994; 3: 245-256.

24 Osman LM, Hyland ME. Patient needs and medication styles in COPD. Eur Respir Rev 2005; 14: 89-92.

25 National Collaborating Centre for Chronic Conditions. Chronic obstructive pulmonary disease. National clinical guideline on management of chronic obstructive pulmonary disease in adults in primary and secondary care. Thorax 2004; 59: Suppl. 1, 1-232. 